\title{
Immediate effect of proprioceptive neuromuscular facilitation on scapular dyskinesia of volleyball players
}

\author{
Fernanda Silvestre Adamatti, William Dhein, Gisele Oltramari Meneghini*.
}

Centro Universitário da Serra Gaúcha - FSG, Caxias do Sul - (RS) Brazil.

\begin{abstract}
Background: Scapular dyskinesia can be caused by several factors, such as poor posture (thoracic kyphosis or cervical hyperlordosis) or changes in the activation and coordination of scapular stabilizing muscles. Therefore, the term is correctly used when there is a change in the scapular biomechanics at rest or during movement. Objective: Evaluate the effects of the proprioceptive neuromuscular facilitation (PNF) on the scapular dyskinesia of volleyball players. Methods: It is a quasi-experimental study with volleyball players. The study sample consisted of 32 individuals of both sexes, aged between 14 and 17 years old, who presented scapular dyskinesia, identified by anamnesis and physical examination. For data collection, a sociodemographic assessment developed by the researchers was used, containing personal data, pain assessment through the Nordic Pain Questionnaire and an image and video record was made for the analysis of photogrammetry and the Scapular Dyskinesia Test. For data analysis, it was adopted a significance level of $5 \%$. Results: Most of the sample $(71.9 \%)$ has practiced volleyball for more than a year, $65.6 \%$ trains three times a week, $84.4 \%$ were attackers, $96.9 \%$ were right-handed and $100 \%$ of the sample presented pain symptoms in the last twelve months. There was a significant difference in the abduction test of both scapulae and in the weight discharge of the right upper limb after the protocol. Conclusion: The PNF proved to be effective in the initial phase of the treatment of scapular dyskinesia acting on neuromuscular balance and scapular orientation.
\end{abstract}

Keywords: Volleyball; Scapula; Cinematics; Physiotherapy.

\section{BACKGROUND}

Volleyball was created in 1895 by the American Willian George Morgan and arrived in Brazil around 1915. Over the years, this sport has been gaining space and has become a very popular team sport worldwide ${ }^{(1)}$. According to Franco et al(2), volleyball is the second most popular sport in Brazil and its practice can occur both recreational and professional. Due to the numerous titles won by Brazilian volleyball, young people and children have been encouraged to start training at an earlier age. Arena and Carazzato(3) report that specific training starts around 10 years of age and is accentuated at $12-13$ years old, when teams start participating in competitions of smaller categories.

For the execution of the game it is necessary to have six players on the court, in specific positions, who perform fundamentals of serve, headline, cut or attack and block. For these functions to be performed correctly and successfully, it is essential that the athlete coordinates a complex pattern of movements, especially in the upper limbs ${ }^{(4)}$. One of the main fundamentals of the game is "cut", which, if successful, awards the point to the team. For the movement to be executed, the athlete must make two long strides and, at the same time, throw the arms back, in the hyperextension movement of the glenohumeral joint and adduction of the scapula. Then, the jump phase, in which the athlete seeks maximum vertical height and, at the same time, does bilateral shoulder flexion to assist in the thrust.

And finally, the attack itself, which occurs when the athlete is positioned to hit the ball. In this phase, the non-dominant arm remains in shoulder flexion and the limb that will perform the cut begins an glenohumeral adduction and extension, until it reaches the ball. The serve movement resembles the attack movement ${ }^{(4)}$. For the attack and serve phases to occur correctly, constant biomechanical alignment of the glenohumeral joint is necessary. When the upper limbs move, the head of the humerus must be centered in the glenoid cavity and the scapular muscles are responsible for this to occur. The humerus and the scapula come into action in order to align forces and generate dynamic balance in the joint. This synergy is called the scapuloumeral rhythm ${ }^{(5)}$.

According to Hamill and Knutzen(6), the joints of the shoulder complex must act concomitantly to coordinate arm movements. In volleyball, to perform the actions of attack and serve, the arm is raised in flexion and abduction and, in order for the athlete to be able to perform the movements in all amplitude, the scapula must enter into action by making a lateral tipper. In addition, the clavicle acts by rotating upwards to allow scapular movement. 
In order for all these movements to be generated to prevent injuries and change kinematics, dynamic stabilization of the muscles inserted in this region is necessary. The scapula is over the serratus anterior and subscapularis muscles, which move transversely to each other as the movement is generated. When the arm is raised, the muscles of the rotator cuff (teres minor, subscapularis, infraspinatus and supraspinatus) act to stabilize the humeral head. The action of the deltoid is equivalent to $50 \%$ of the muscular strength for shoulder flexion and abduction. However, as the force vector of the deltoid muscle is vertical, it is the supraspinatus that performs the first degrees of movement, which further compresses the humeral head against resistance so that there is no impact of it with the acromion ${ }^{(6)}$.

However, when there is a change in this movement or in the positioning of the scapula, the terms commonly used are "winged scapula" or, more appropriately, "scapular dyskinesia". Winged scapula refers to the prominence of the medial edge of the scapula, but this terminology fails to identify if the origin of the change is static, dynamic or both. Scapular dyskinesia is a general term, defined by Kliber and Sciascia(7), which refers to any unbalanced movement of the scapula during movements of the upper limbs or in static posture.

Scapular dyskinesia can be caused by several factors, such as poor posture (thoracic kyphosis or cervical hyperlordosis) or changes in the activation and coordination of the scapular stabilizing muscles. Therefore, the term is correctly used when there is a change in the scapular biomechanics at rest or during movement ${ }^{(8)}$.

Several tests have been developed to analyze scapular dyskinesis objectively. Kliber et $\mathrm{al}^{(9)}$, classified dyskinesia as: type I: prominence of the lower angle of the scapula; type II: prominence of the medial edge of the scapula; type III: upper edge of the stable scapula remains elevated; and type IV: asymmetry between the shoulder blades. One of the possible contributions to the development of scapular dyskinesia is the instability of the scapulothoracic muscles, which leads to abnormal scapular positioning and alters the scapularhumeral rhythm. The serratus anterior and the lower trapezius are the muscles that most contribute to the upward rotation of the scapula and acromial elevation, movements that allow flexion and abduction of the arm for the fundamentals of volleyball. If, for some reason, part of these muscular forces are altered, the scapular movement becomes abnormal ${ }^{(10)}$.

As treatment alternatives for scapular dyskinesia, it is suggested exercises that recruit muscles that stabilize and move the scapula. One of these alternatives would be the Proprioceptive Neuromuscular Facilitation (PNF). This technique was developed by Herman Kabat together with physiotherapists Margareth Knott and Doroty Voss and aims the training with activities to facilitate, inhibit, relax and strengthen of muscle groups ${ }^{(11,12)}$.

One of the benefits of the PNF technique is the manual contact of the therapist, as it stimulates the skin receptors and information to the patient in the correct direction to resist movement. In addition, other benefits that contact brings are the stimulation of the contraction of synergistic muscles to reinforce the movement, promotion of stabilization in the axis and the intensification of the contraction caused by the pressure made on the muscle ${ }^{(13)}$.

Within the PNF there are specific techniques that use isotonic, concentric and eccentric, and isometric contractions. The reversal of stabilizers is a technique where the patient starts by contracting the agonist muscles and later the antagonists, without pausing or relaxing. To perform the method, the therapist applies a manual resistance force and request that the patient oppose the movement. Approach and traction actions are used, in short movements, in order to increase scapular stability ${ }^{(13)}$.

Therefore, in view of the implications found in scapular dyskinesia, a physiotherapeutic intervention becomes essential to prevent and treat the asymmetries found, especially in volleyball, in which the alterations can compromise the biomechanical alignment of the scapulohumeral rhythm and impair the athletes' performance. Thus, this study aims to analyze the effects of PNF on scapular dyskinesia of volleyball players.

\section{METHODS \\ Study Design}

This is a quantitative study with a semiexperimental design. The study was developed guaranteeing compliance with the Resolution 466/12 or 510/16 of the National Health Council, and was approved by the Research Ethics Committee - FSG with no 2.661.223.

\section{Sample}

The sample consisted of 32 volleyball players, of both genders, between 14 and 17 years old, belonging to a volleyball school in the city of Caxias do Sul, Rio Grande do Sul. The sampling was by convenience and the eligibility criteria resulted in the participation of volleyball school players who were positive in the Scapular dyskinesis test ${ }^{(14)}$. There was no exclusion criteria.

\section{Procedures}

All subjects were assessed at the Madre Imilda volleyball school, in a room according to the availability of practitioners. Initially, the participants signed the informed consent form (IC), which contained the objectives and procedures of the study respecting all 
ethical aspects according to the resolution 466/12. The subjects answered an evaluation form (age, education, physical activities they develop, history of pain/discomfort, frequency of training, intensity of training, among others). After that, the subjects were submitted to an evaluation of pain/discomfort (Nordic Questionnaire), physical examination (Scapular dyskinesis test; computerized photogrammetry; weight unloading), later, to an intervention (proprioceptive neuromuscular facilitation) and reassessment of the variables from the physical examination.

After completing the initial assessment form, the practitioner was asked about the complaints of pain/discomfort (Nordic Pain Questionnaire) due to the following questions: "In the last 12 months, did you have problems (pain, tingling/numbness) in specific regions of the body (neck, shoulder, elbow, wrist and hand, dorsal, cervical, lumbar spine, hips, thighs and buttocks, knees, ankles and feet)?"; "In the past 12 months, have you been prevented from performing normal activities (work, domestic and leisure activities) because of this problem in the regions mentioned above"; "In the last 12 months, did you consulted a health professional (e.g. doctor, physiotherapist) because of this condition?"; "In the past seven days, have you had any problems (pain, tingling/numbness) in the body regions". After completing the questionnaire, the subject started the physical examination.

The Scapular Dyskinesis Test ${ }^{(14)}$ was used to assess the presence/absence of scapular dyskinesia. In addition, it seeks to classify it into: (1) haul or (2) dysrhythmia; (a) unilateral; (b) bilateral and (I) obvious (II) smooth. To perform the test, male subjects were asked to remove their shirt and female participants to wear the top of a bikini in order to better observe the scapular kinematics (Figure 1). Classifications were given by analyzing scapular bony prominences such as upper scapular angle, lower scapular angle and distance from the medial edge of the scapula in relation to the spinous processes of the thoracic spine.

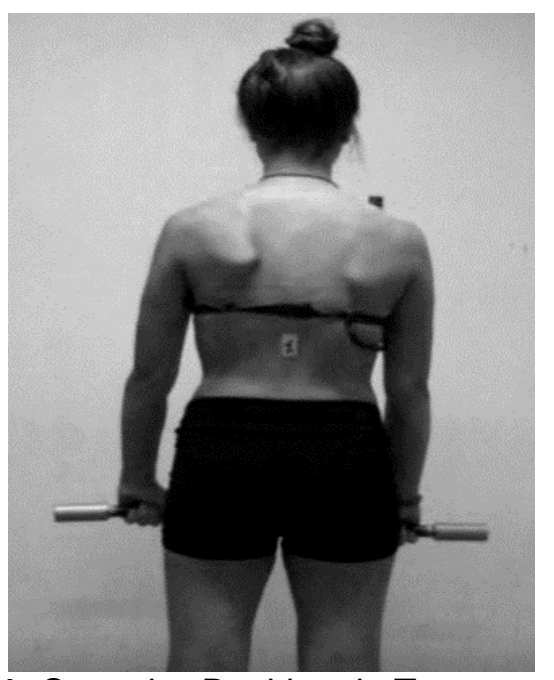

Figure 1. Scapular Dyskinesis Test.
During the test, a camera was used, arranged on an adjustable tripod, to record the movements of the scapular posterior view. The subject was instructed to remain in orthostasis, 2 to 3 meters from the camera. After familiarization, the subject was asked to perform five bilateral repetitions of the active shoulder flexion and abduction movements. It is recommended to use an external load determined according to the subjects' body weight. Resulting in $1.4 \mathrm{~kg}$ for those weighing less than $68.1 \mathrm{~kg}$ and 2.3 $\mathrm{kg}$ for those weighing $68.1 \mathrm{~kg}$ or more ${ }^{(14)}$.

After the test, points were marked on the bony prominences of the cervical spine and scapula ${ }^{(15,16)}$ to assess the static scapular position using computerized photogrammetry (Figure 2). The anatomical references for demarcation in the posterior view were the bony prominences of the $\mathrm{C} 7$ spinous process, the upper and lower scapular angles and their corresponding spinous processes, T2 and T7 respectively ${ }^{(15,16)}$. With the evaluation of photogrammetry, measurements of scapular postural changes were obtained, such as elevation or haul.

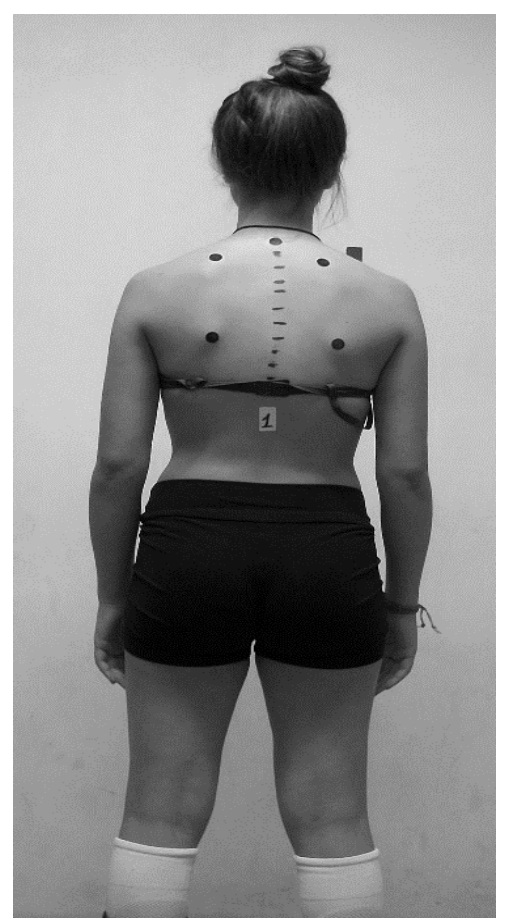

Figure 2. Anatomical references of computerized photogrammetry.

Then, the verification of weight unloading (Figure 3) of the upper limbs was performed using two digital scales G-Life Slim, previously calibrated so that there was no measurement bias. They were placed on the floor, side by side. The subject was asked to stay in a position of four supports and to place each hand on top of each scale ${ }^{(17)}$. Images of the subjects in position of four supports were also taken to assess the scapulae as weight was released on the upper limbs. 


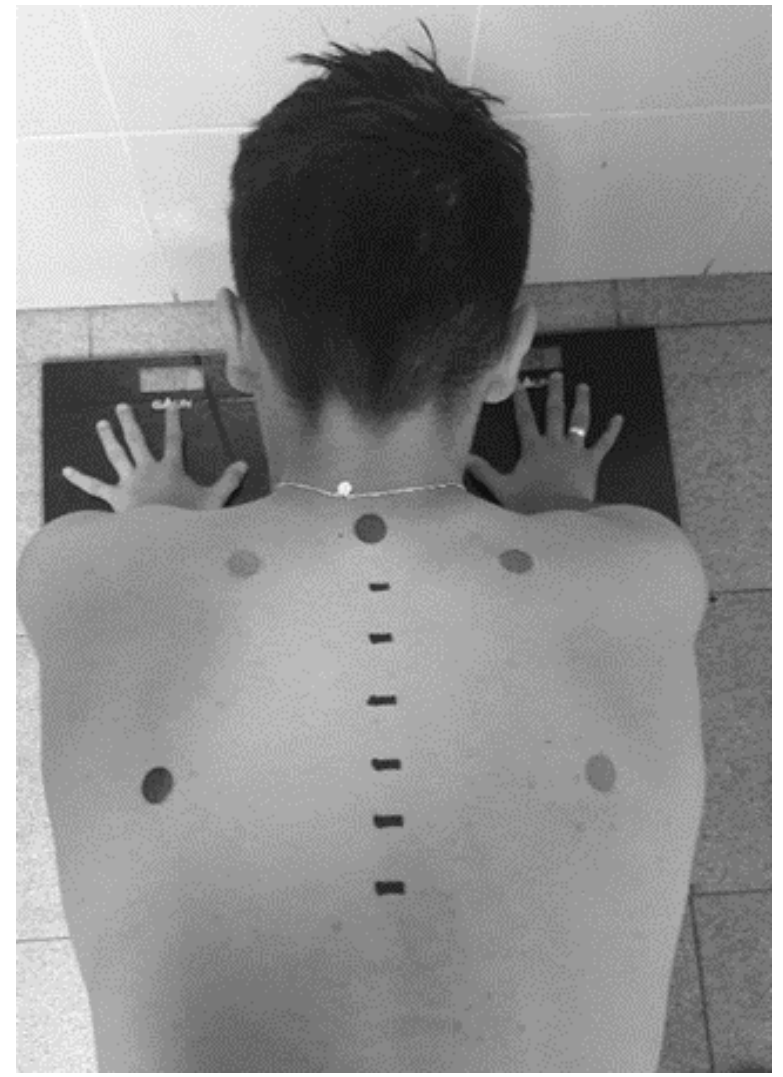

Figure 3. Unloading weight on the digital scale

\section{Intervention}

After physical examinations, the PNF exercise protocol was initiated. The subject was placed in lateral decubitus and ten repetitions of scapular movement patterns (antero-elevation; posterodepression; antero-depression and posteroelevation) were performed bilaterally using the PNF rhythmic initiation technique. Then, the subject was in the position of four supports and the technique of reversing the stabilizers of the PNF was performed, in which the objective was to recruit the scapular stabilizing muscles as scapular lift, upper trapezius, middle trapezius, lower trapezoid, rhomboids and serratus anterior. Repetitions were performed until 5 minutes were completed for each scapula. One of the proposals of this method was to obtain muscle contraction through a principle called irradiation or cross education, in which the application of several techniques is used in order to strengthen muscles and gain joint stability ${ }^{(13)}$. After applying the PNF protocol, subjects were reevaluated using the same instruments used in the assessment.

\section{Statistical analysis}

A statistical analysis was performed using SPSS v20.0 software. Initially, they were verified as normal data using the Kolmogorov-Smirnov test. After the normal distribution was confirmed (parametric data), the scalar data were performed using descriptive statistics (mean, standard deviation, maximum and standard) and compared in situations using the dependent/paired t test.

Categorical data are presented by means of absolute frequencies ( $\mathrm{n}$ and percentage) and compared using the Wilcoxon signed-rank test $(Z)$. The level of significance adopted was $5 \%$.

\section{RESULTS}

The sample included 32 subjects, 24 (75\%) female and $8(25 \%)$ male. Most of the sample $(71.9 \%)$ has practiced volleyball for more than a year and $65.6 \%$ have been training three times a week. The positions of lifter, attacker and libero were present, with the attacker being the most prevalent, $84.4 \%$ of the subjects. Most subjects did not practice any other physical activity (56.3\%) and 31 of them $(96.9 \%)$ were right-handed and 1 (3.1\%) lefthanded, as shown in Table 1.

In relation to the places of frequent pain, Table 1 illustrates that $65.62 \%$ of the sample has frequent pain symptoms distributed in different body regions. Regarding pain sites in the last 12 months, $100 \%$ of the participants had pain symptoms, where $43.75 \%$ of them were in the upper limbs (upper limbs), trunk and lower limbs (lower limbs), more specifically in the shoulders and knees. Still in Table 1, it is possible to observe that $75 \%$ of the subjects had pain in the last seven days, more localized in upper limbs, trunk and lower limbs, and the majority of the sample population (59.3\%) did not seek health professionals to treat pain symptoms.

Table 2 shows the results of the Scapular Dyskinesia tests and the analysis of Computerized Photogrammetry, in which it was observed that $84.4 \%$ of the subjects presented scapular dyskinesia in both scapulae in the flexion test and $59.4 \%$ presented dyskinesia in both scapulae in the abduction test.

Through the data shown in Table 3, in relation to the outcomes of computerized photogrammetry and scale, it can be seen that the comparison between weight unloading on the scale before and after the right side showed statistical significance $(p$ $<0.001$ ), meaning that after the intervention with the PNF, the subjects showed an improvement in weight unloading on the right scapula.

Regarding the Scapular Dyskinesia Test, Table 4 shows significant comparisons between the abduction test of the right scapula before and after $(p<0.46)$ and between the abduction test of the left scapula before and after ( $p<0.008)$, based on the analysis of the Wilcoxon Signed-Ranks Test, based on negative ranks. This means that both the right scapula and the left scapula showed variations in the dyskinesia test, that is, before the PNF protocol the majority had dyskinesia and after the protocol a smaller number of subjects had dyskinesia. 
Table 1 - Socio-demographic and clinical characteristics of the studied population

\begin{tabular}{|c|c|}
\hline Variable & $\mathrm{n}=32(100 \%)$ \\
\hline \multicolumn{2}{|l|}{ Gender } \\
\hline Male & $8(25 \%)$ \\
\hline Female & $24(75 \%)$ \\
\hline \multicolumn{2}{|l|}{ Volleyball Time } \\
\hline Less than 6 months & $3(9.4 \%)$ \\
\hline 6 months to 1 year & $6(18.8 \%)$ \\
\hline 1 to 3 years & $11(34.4 \%)$ \\
\hline Over 3 years & $12(37.5 \%)$ \\
\hline \multicolumn{2}{|l|}{ Volleyball Frequency } \\
\hline 2 times per week & $2(6.3 \%)$ \\
\hline 3 times per week & $21(65.6 \%)$ \\
\hline 4 times per week & $3(9.4 \%)$ \\
\hline 5 times per week & $2(6.3 \%)$ \\
\hline 6 times per week & $4(12.5 \%)$ \\
\hline \multicolumn{2}{|l|}{ Position } \\
\hline Lifter & $2(6.3 \%)$ \\
\hline Attacker & $27(84.4 \%)$ \\
\hline Libero & $3(9.4 \%)$ \\
\hline \multicolumn{2}{|l|}{ Practices other physical activity } \\
\hline Yes & $14(43.7 \%)$ \\
\hline No & $18(56.3 \%)$ \\
\hline \multicolumn{2}{|l|}{ Dominance } \\
\hline Right-handed & $31(96.9 \%)$ \\
\hline Left-handed & $1(3.1 \%)$ \\
\hline \multicolumn{2}{|l|}{ Frequent pain site } \\
\hline Neck & $1(3.1 \%)$ \\
\hline Shoulders & $5(15.6 \%)$ \\
\hline Upper Back & $3(9.37 \%)$ \\
\hline Lower back & $6(18.7 \%)$ \\
\hline Fists/hands & $1(3.1 \%)$ \\
\hline Knees & $5(15.6 \%)$ \\
\hline Ankles/feet & $5(15.6 \%)$ \\
\hline Painless & $11(34.3 \%)$ \\
\hline \multicolumn{2}{|l|}{ Pain site in the last 12 months } \\
\hline Upper limbs only & $1(3.1 \%)$ \\
\hline Trunk only & $1(3.1 \%)$ \\
\hline Lower limbs only & $1(3.1 \%)$ \\
\hline Upper limbs + Lower limbs & $7(21.9 \%)$ \\
\hline Upper limbs + Trunk & $4(12.5 \%)$ \\
\hline Upper limbs + Trunk + Lower limbs & $14(43.75 \%)$ \\
\hline Trunk + Lower limbs & $4(12.5 \%)$ \\
\hline \multicolumn{2}{|l|}{ Pain site in the last 7 days } \\
\hline Upper limbs only & $6(18.7 \%)$ \\
\hline Trunk only & $6(18.7 \%)$ \\
\hline Lower limbs only & $6(18.7 \%)$ \\
\hline Upper limbs + Lower limbs & $2(6.25 \%)$ \\
\hline Upper limbs + Trunk & $3(9.38 \%)$ \\
\hline Upper limbs + Trunk + Lower limbs & $1(3.12 \%)$ \\
\hline Painless & $8(25 \%)$ \\
\hline \multicolumn{2}{|l|}{$\begin{array}{l}\text { Sought health professional to treat pain } \\
\text { Yes }\end{array}$} \\
\hline No & $13(40.6 \%)$ \\
\hline & $19(59.3 \%)$ \\
\hline
\end{tabular}


Effect of PNF on scapular dyskinesia

Table 2. Results of scapular dyskinesia and computerized photogrammetry tests

\begin{tabular}{|c|c|c|c|c|}
\hline & \multicolumn{2}{|c|}{ Right } & \multicolumn{2}{|c|}{ Left } \\
\hline & Before & After & Before & After \\
\hline \multicolumn{5}{|c|}{ Scap Dysk Test Flexion } \\
\hline Normal scapulae & $5(15.6 \%)$ & 7 (21.9\%) & $5(15.6 \%)$ & $8(26 \%)$ \\
\hline Dyskinesia & $27(84.4 \%)$ & $25(78.1 \%)$ & $27(84.4 \%)$ & $24(75 \%)$ \\
\hline \multicolumn{5}{|c|}{ Scap Dysk Test Abduction } \\
\hline Normal scapulae & $13(40.6 \%)$ & $17(53.1 \%)$ & $13(40.6 \%)$ & $13(40.6 \%)$ \\
\hline Dyskinesia & $19(59.4 \%)$ & $15(46.9 \%)$ & $19(59.4 \%)$ & $19(59,4 \%)$ \\
\hline \multicolumn{5}{|l|}{ Photogrammetry } \\
\hline Symmetrical & $13(40.6 \%)$ & $13(40.6 \%)$ & $6(18.8 \%)$ & $12(37.5 \%)$ \\
\hline Asymmetric & $19(59.4 \%)$ & $19(59.4 \%)$ & $26(81.2 \%)$ & $20(62.5 \%)$ \\
\hline
\end{tabular}

Table 3. Comparisons between the outcomes of computerized photogrammetry and scale.

\begin{tabular}{|c|c|c|c|c|}
\hline Variable & Average & $\mathrm{n}=32$ & $\mathrm{SD} \pm$ & $P$ value \\
\hline Right upper angular distance of scapular spine - Before & $12.0006 \mathrm{~cm}$ & 32 & 2.31731 & .932 \\
\hline Right upper angular distance of scapular spine - After & $11.9775 \mathrm{~cm}$ & 32 & 2.58259 & \\
\hline Right lower angular distance of scapular spine - Before & $13.0516 \mathrm{~cm}$ & 32 & 2.75856 & .667 \\
\hline Right lower angular distance of scapular spine - After & $13.1656 \mathrm{~cm}$ & 32 & 2.85039 & \\
\hline Left upper angular distance of scapular spine - Before & $11.3650 \mathrm{~cm}$ & 32 & 2.18227 & .672 \\
\hline Left upper angular distance of scapular spine - After & $11.2528 \mathrm{~cm}$ & 32 & 2.54322 & \\
\hline Left lower angular distance of scapular spine - Before & $13.8269 \mathrm{~cm}$ & 32 & 2.39942 & .715 \\
\hline Left lower angular distance of scapular spine - After & $13.7066 \mathrm{~cm}$ & 32 & 2.67238 & \\
\hline Scale - RUL Before & $10.5500 \mathrm{~kg}$ & 32 & 3.43070 & $.001^{*}$ \\
\hline Scale - RUL After & $11.7250 \mathrm{~kg}$ & 32 & 3.36145 & \\
\hline Scale - LUL Before & 11.1344 kg & 32 & 3.17303 & .114 \\
\hline Scale - LUL After & 11.8219 kg & 32 & 3.76561 & \\
\hline
\end{tabular}

Table 4. Comparison between the outcomes of the Scapular Dyskinesia Test

\begin{tabular}{lcc}
\hline Variable & $\mathrm{n}=32$ & P value \\
\hline Right Flexion - Scapular Dyskinesia Test - Before \& After & 32 & .157 \\
Right Abduction - Scapular Dyskinesia Test - Before \& After & 32 & $.046^{\star}$ \\
Left Flexion - Scapular Dyskinesia Test - Before \& After & 32 & .083 \\
Left Abduction - Scapular Dyskinesia Test - Before \& After & 32 & $.008^{*}$ \\
\hline
\end{tabular}




\section{DISCUSSION}

In the present study, it was found that $75 \%$ of the population was female and had an average young age. Nowadays, stimulating the practice of sports in childhood and adolescence has become more and more common, and private clubs or local sports departments promote training and provide incentives to more and more athletes ${ }^{(18)}$. Furthermore, adolescents have a different energy requirement than adults and, thus, the practice of physical activities influences the achievement of desired movement patterns and optimizes bone mass gain during adolescence $^{(19,20)}$.

As for the time of practicing volleyball, $71.9 \%$ of the sample answered that they have practiced the sport for more than a year and $65.6 \%$ practice three times a week. Mesquita et $\mathrm{al}^{(20)}$, conducted a study with girls from 13 to 17 years old who practiced volleyball, whose objective was to observe the bone mineral density of the athletes and to compare it with a control group. In the volleyball group studied in the present study, the frequency of three weekly sessions was observed, in which most of the participants had been training for three or four years and it can be seen that volleyball is recommended, because the muscular and mechanical overload of the sport enhances peak bone mass. It was observed in another study, that the places where there is more mechanical overload in the practice of volleyball are the shoulders and the shoulder blades, since the synergy between these two structures allows the athlete to be able to perform the fundamentals required by the sport ${ }^{(5)}$

During the practice of volleyball, athletes are subject to several intrinsic and extrinsic factors that can influence their performance. Pain is the main symptom reported by athletes and can lead to a decrease in physical performance and possible withdrawal ${ }^{2}$. In the present study, $100 \%$ of the participants reported pain in some part of the body in the last twelve months, $43.75 \%$ of which had symptoms in upper limbs, trunk and lower limbs, more specifically in the shoulders, upper back and knees. Anza et $\mathrm{al}^{(21)}$, conducted a study with children and youth volleyball athletes using the Nordic Musculoskeletal Questionnaire to assess pain symptoms and $52.2 \%$ of the participants had complaints about the shoulders in the same category, corroborating the results found in the present study.

In order for the athlete to be able to efficiently perform the main foundation of volleyball, the attack, it is necessary that the movements between the scapula and the humerus are coordinated, the so-called scapuloumeral rhythm. When there is any factor, be it trauma, structural issues or fatigue, that changes the scapular biomechanics, scapular dyskinesia appears $^{(22)}$. In the present study, $84.4 \%$ of the participants had dyskinesia in both scapulae in the flexion test and $59.4 \%$ had dyskinesia in both scapulae in the abduction test. In a study by Pluim ${ }^{(23)}$, the majority of asymptomatic athletes showed changes in the dynamic control of the scapula and $67 \%$ to $100 \%$ of them presented this change associated with shoulder injuries. In another study, with 142 athletes, $26 \%$ had dyskinesia on the right scapula, $36.6 \%$ on the left scapula and $22.5 \%$ on both scapulae ${ }^{(15)}$. Burn et $\mathrm{al}^{(24)}$ carried out a survey on the sports that the athletes used the upper limbs above the head, characterized by them as "aerial athletes" and the most common sport was volleyball, comprising $30 \%$ of the sample. In addition, $54.5 \%$ of aerial athletes had scapular dyskinesia.

Scapular dyskinesia is an alteration that can compromise the performance of athletes or subjects who use the scapula as the main training structure. Thus, treating scapular dyskinesia is essential for any physiotherapist who works directly with these subjects. As a treatment alternative, neuromuscular reeducation should be taken into account, as these exercises reorganize and guide the scapular kinematics and must be prescribed by physiotherapists. Proprioceptive Neuromuscular Facilitation (PNF) should be used as the first technique in the rehabilitation process to promote isolated scapular movement and motor learning ${ }^{(25)}$. Schory et al(26), performed a systematic review using electromyography to assess the activity of the upper trapezius compared to the middle trapezius, lower trapezius or serratus anterior in eccentric exercises in the frontal and sagittal planes and concluded that lowload exercises are effective in neuromuscular organization and scapular orientation.

The stabilizer reversion technique used in the present study allows the orientation and muscle activation of scapular stabilizers through manual contact by the therapist, which stimulates agonist and antagonist muscles and allows the correct muscle recruitment time, as exercise programs high resistance reduce neuromuscular control that leads to accentuated kinematic dysfunction and do not provide pain relief to the patient ${ }^{(27)}$.

Cools et $\mathrm{al}^{(28)}$, bring in their study the importance of identifying which deficiencies are leading to scapular dyskinesia and that the rehabilitation should be geared to the functional demands of each subject. Thus, in volleyball, the focus of the treatment is with exercises for muscle control of the scapula and the gain of strength during the specific movements of the sport. In another study, an algorithm for the clinical use of techniques for the treatment of scapular dyskinesia was published, which states that the focus of rehabilitation for athletes should be focused on muscle control, which occurs primarily through the cocontraction of agonist and antagonists muscles that stabilize the scapula and, later, in the muscular strength gain, which combined will generate greater control and resistance so that the athlete is able to perform the sporting gestures with efficiency ${ }^{(29)}$. 
In the present study, there was a statistically significant difference in weight unloading on the right side, measured using a scale, after applying the PNF technique. According to Cools et al ${ }^{(28)}$, the awareness of muscle control provided by the stabilizer reversal technique, facilitated by the therapist, gives the patient active control and scapular orientation, generating proprioception and normalizing the scapular position, especially on the dominant side, which the subject unloads more weight due to having greater motor control and muscle activation.

Regarding the scapular dyskinesia test, it was also observed in the present study that there was a statistically significant difference in the abduction test before and after in both scapulae, implying a reduction in both dyskinesia. In Kliber's study et al(30), an exercise protocol was used to control the scapula and, after electromyographic analysis, a significant interaction between the exercises and the muscles can be seen, indicating that certain exercises activate specific muscles in greater proportion than other muscle groups. It also demonstrated that moderate muscle activation is adequate for neuromuscular control of the scapula and ideal for the initial phase of rehabilitation. In addition, they report that cocontraction allows muscle training, which keeps the muscles organized, generating proprioceptive rehabilitation and guidance on scapular kinematics, which explains the results found in the present study.

\section{CONCLUSION}

It is concluded, from this study, that scapular dyskinesia is vigorously present in volleyball practitioners and, even if asymptomatic, the treatment should be aimed at neuromuscular balance, with moderate muscle activation, providing a rebalancing in scapular kinematics. Therefore, the PNF proved to be an effective technique for the initial phase of rehabilitation, causing, in the present study, a statistically significant difference in weight unloading on the scale after application and in the scapular abduction test before and after in both scapulae.

Thus, the relevance of this study is extremely important for the physiotherapy, since knowledge about the biomechanical changes that dyskinesia causes is of utmost importance so that the best exercises in the rehabilitation process are listed. It is suggested that further research be developed in order to prove the effectiveness of new techniques for neuromuscular balance in scapular dyskinesia.

\begin{abstract}
Authors' contributions: FSA: development of the study, data collection, data analysis, discussion and article writing; WD: development of the study and data analysis; GOM: Adviser, development of the study and discussion of the article.
\end{abstract}

Financial support: The authors declare that there was no financial support.
Conflict of interest: The authors declare that there was no conflict of interests.

\section{REFERENCES}

1. Bizzocchi C. O voleibol de alto nível: da iniciação à competição. Barueri:Manole; 2013.

2. Franco JB, Lucheta PA, Teixeira LS. Projeto para prevenção de lesões do ombro em atletas de voleibol. Ensaios e Ciência: C Biológicas, Agrárias e da Saúde. 2011;15(1):33-44.

3. Arena SS, Carazzato JG. A relação entre o acompanhamento médico e a incidência de lesões esportivas em atletas jovens de São Paulo. Rev Bras Med Esporte. 2007;13(4):217-21.

4. Carnaval PE. Cinesiologia aplicada aos esportes. Rio de Janeiro:Sprint; 2000.

5. Nascimento LR, Bittencourt NFN, Resende RA, Teixeira-Salmela LF, Fonseca ST. Biomecânica aplicada ao voleibol: análise do complexo do ombro e implicações para avaliação e desempenho. Ter Man. 2010;8(40):483-90.

6. Hamill J, Knutzen KM. Bases biomecânicas do movimento humano. Barueri:Manole; 2012.

7. Kliber WB, Sciascia A. Current concepts: scapular dyskinesis. Br J Sports Med. 2010;44(5):300-5.

8. Burkhart SS, Morgan CD, Kliber B. The disabled throwing shoulder: spectrum of pathology part I: pathoanatomy and biomechanics. Arthroscopy. 2003;19(4):404-20.

9. Kliber WB, Uhl TL, Maddux JW, Brooks PV, Zeller B, Mcmullen J. Qualitative clinical evaluation of scapular dysfunction: a reliability study. J Shoulder Elbow Surg. 2002;11(6):550-56.

10. Paine R, Voight ML. The role of the scapula. Int $J$ Sports Phys Ther. 2013;8(5):617-29.

11. Pinheiro HA. Efeito da facilitação neuromuscular proprioceptiva no equilíbrio de indivíduo com degeneração espinocerebelar recessiva. Fisioter Bras. 2012;13(2):137-41.

12. Voight ML, Thomson BC. The role of the scapula in the rehabilitation of shoulder injuries. J Athl Train. 2000;35(3):364-72.

13. Adler SS, Beckers D, Buck M. PNF: facilitação neuromuscular proprioceptiva: um guia ilustrado. Barueri:Manole; 2007.

14. McClure P, Tate AR, Kareha S, Irwin D, Zlupko E. A clinical method for identifying scapular dyskinesis, part 1: reability. $J$ Athl Train. 2009;44(2):160-4.

15. Guariglia DA, Pereira LM, Pereira HM, Cardoso JR. Avaliação da confiabilidade e usabilidade de três diferentes programas computacionais para a análise fotogramétrica do ângulo de flexão do quadril. Fisioter Pesq. 2011;18(3):247-51.

16. Polisseni MLC, Resende CP, Faião DR, Ferreira MEC, Fontes LS. Avaliação postural e muscular da cintura escapular em adultos jovens, estudantes universitários. R Bras Ci e Mov. 2010;18(3):56-63. 
17. Bohannon RW, Larkin PA. Lower extremity weight bearing under various standing conditions in independently ambulatory patients with hemiparesis. Phys Ther. 1985;65(9):1323-5.

18. Tomazoni SS, Zanetto BGS, Leal Junior ECP. Prevalência de lesões musculoesqueléticas em atletas adolescentes. ConScientiae Saúde. 2011;10(1):122-28.

19. Silva JA. A prática de voleibol na escola: investigação sobre a relação ensino aprendizagem das habilidades básicas do voleibol [monografia]. Duas Estradas: Universidade de Brasília; 2014.

20. Mesquita WG, Fonseca RMC, França NM. Influência do voleibol na densidade mineral óssea de adolescentes do sexo feminino. Rev Bras Med Esporte. 2008;14(6):500-3.

21. Anza R, Denis M, Silva MF. Análise da aptidão física, da antropometria e da prevalência de sintomas osteomusculares na categoria infantojuvenil do voleibol. Rev Bras Med Esporte. 2013;19(1):62-5.

22. Kliber WB, Ludewig PM, McClure PW, Michener LA, Bak K, Sciascia AD. Clinical implications of scapular dyskinesis in shoulder injury: the 2013 consensus statement from the 'scapular summit'. Br J Sports Med. 2013; 47:877-85.

23. Pluim BM. Scapular dyskinesis: practical applications. Br J Sports Med. 2013;47(14):875-6.
24. Burn MB, McCulloch PC, Lintner DM, Liberman $\mathrm{SR}$, Harris JD. Prevalence of scapular dyskinesis in overhead and nonoverhead athletes. Orthop $\mathrm{J}$ Sports Med. 2016;4(2):1-8.

25. McClure P, Greenberg E, Kareha S. Evaluation and management of scapular dysfunction. Sports Med Arthrosc Rev. 2012;20(1):39-48.

26. Schory A, Bidinger E, Wolf J, Murray L. A systematic review of the exercises that produce optimal muscles ratios of the scapular stabilizers in normal shoulders. Int $\mathrm{J}$ Sports Phys Ther. 2016;11(3):321-36.

27. Ludewig PM, Reynolds JF. The association of scapular kinematics and glenohumeral joint pathologies. J Orthop Sports Phys Ther. 2009;39(2):90-104.

28. Cools AMJ, Struyf F, Mey KD, Maenhout A, Castelein B, Cagnie B. Rehabilitation of scapular dyskinesis: from the office work to the elite overhead athlete. $\mathrm{Br} J$ Sports Med. 2014;48(8):692-7.

29. Ellenbecker TS, Cools A. Rehabilitation of shoulder impingement syndrome and rotator cuff injuries: an evidence-based review. $\mathrm{Br} \mathrm{J}$ Sports Med. 2010;44(5):319-27.

30. Kliber WB, Sciascia AD, Timothy LU, Tambay N, Cunningham T. Electromyographic analysis of specific exercises for scapular control in early phases of shoulder rehabilitation. Am J Sports Med. 2008;36(9):1789-98. 